\title{
Cryptococcosis by Cryptococcus neoformans/Cryptococcus gattii Species Complexes in non-HIV-Infected Patients in Southeastern Brazil
}

\author{
Erika Nascimento[1], Patrícia Helena Grizante Barião ${ }^{[2]}$, Marcia Regina von Zeska Kress ${ }^{[2]}$, \\ Fernando Crivelenti Vilar ${ }^{[1]}$, Rodrigo de Carvalho Santana ${ }^{[1]}$, Gilberto Gambero Gaspar ${ }^{[1]}$ \\ and Roberto Martinez ${ }^{[1]}$
}

[1]. Universidade de São Paulo, Faculdade de Medicina de Ribeirão Preto, Departamento de Clínica Médica, Ribeirão Preto, SP, Brasil.

[2]. Universidade de São Paulo, Faculdade de Ciências Farmacêuticas de Ribeirão Preto,

Departamento de Análises Clínicas, Toxicológicas e Bromatológicas, Ribeirão Preto, SP, Brasil.

\begin{abstract}
Introduction: The clinical manifestations of cryptococcosis are usually associated with the infecting agents Cryptococcus neoformans $(\mathrm{CN})$ and $C$. gattii $(\mathrm{CG})$ species complexes and the host. In this study, non-HIV-infected patients, at a university hospital in southeastern Brazil, had epidemiological and clinical data associated with cryptococcal disease and isolated Cryptococcus species: CN - 24 patients and CG - 12 patients. Methods: The comparison was comprised of demographic data, predisposing factors, clinical and laboratory manifestations, and outcomes of cryptococcosis patients treated between 2000 and 2016. Immunocompetent and immunosuppressed patients were also compared, irrespective of the infecting species. Cryptococcus spp. were genotyped by PCR-RFLP analysis of the URA5 gene. Results: Infections by the CN species complex (100\% VNI genotype) were associated with drug immunosuppression and fungemia, and patients infected with the CG species complex (83\% VG II and 17\% VGI genotypes) had more evident environmental exposure and higher humoral response. CN and CG affected patients with or without comorbidities. Conclusions: Diabetes mellitus, other chronic non-infectious diseases, and alcoholism were likely predisposing factors for infection by both $\mathrm{CN}$ and $\mathrm{CG}$ species. Immunocompetent patients, independent of the infecting Cryptococcus species complexes, showed a higher occurrence of meningitis and a trend toward less fungal dissemination and longer survival than immunosuppressed hosts.
\end{abstract}

Keywords: Cryptococcal disease. Cryptococcal meningitis. Cryptococcus neoformans complex. Cryptococcus gattii complex.

\section{INTRODUCTION}

In the last two decades, there has been a decline in the occurrence of opportunistic cryptococcosis in AIDS cases with a simultaneous increase in the incidence of cryptococcal disease in non-HIV-infected patients ${ }^{1,2}$. HIV seronegative patients infected with Cryptococcus spp. are a heterogeneous population that includes cases of therapeutic immunosuppression, comorbidities, solid organ transplantation, and immunocompetent individuals with no apparent comorbidity. Differences in the clinical characteristics and lethality

\footnotetext{
Corresponding author: Erika Nascimento. e-mail: erika.nascimento@gmail.com

(D) https://orcid.org/0000-0002-4141-7414

Received 26 April 2021

Accepted 16 July 2021
}

of cryptococcal disease have been observed between these groups of patients and with cryptococcosis associated with AIDS 3 .

Cryptococcal disease is caused predominantly by species of $C$. neoformans $(\mathrm{CN})$ and $C$. gattii (CG) complexes. The $\mathrm{CN}$ complex includes the species $C$. neoformans (genotype VNI/VNII/ VNB), C. deneoformans (genotype VNIV), and a hybrid species (genotype VNIII). CG complex includes $C$. gattii strictu senso, C. deuterogattii, C. bacillisporus, C. tetragattii, and C. decagattii, respectively, genotypes VGI, VGII, VGIII, VGIV, and VGV/IIIc; hydrids between species of the two Cryptococcus complexes have been reported ${ }^{5}$. $\mathrm{CN}$ is more prevalent and has a wider geographical distribution, while CG is more isolated in tropical and subtropical regions, although species of this complex have also been isolated in temperate climate environments ${ }^{6}$. CG is often associated with infections in immunocompetent individuals, in addition to more frequent lung and brain parenchyma lesions ${ }^{7,8}$. Different 
geographical areas may show differences in the predominant genotype/species of Cryptococcus spp. and eventually in the clinical presentation of cryptococcal disease ${ }^{9}$. C. neoformans molecular type VNI is the major agent of cryptococcal disease in Brazil, followed by $C$. gattii, and the prevalence of this last species increases from the southern to northern region of the country ${ }^{6}$.

This study aimed to assess the characteristics of cryptococcosis in non-HIV-infected patients in southeastern Brazil. The clinical aspects of cryptococcal disease in immunocompetent individuals and the comparison of cases with isolation of $\mathrm{CN}$ and $\mathrm{CG}$ species complexes were analyzed. This study has clinical relevance because of the scarcity of studies on cryptococcosis comparing $\mathrm{CN}$ or CG complex infections in non-HIV-infected patients, including immunocompetent individuals, from Brazil.

\section{METHODS}

This retrospective study analyzed the clinical and epidemiological data of non-HIV-infected patients with cryptococcal disease. The patients received medical assistance between 2000 and 2016 at the University Hospital of the Ribeirão Preto Medical School, University of São Paulo (SP), and lived in the region of Ribeirão Preto, SP, Brazil. The data were analyzed according to the results of the Cryptococcus spp. genotyping, which were divided into two groups: 1) the $\mathrm{CN}$ group with 24 patients infected with species of the C. neoformans complex; and 2) the CG group with 12 patients infected with species of the C. gattii complex. Further analysis compared data from immunocompetent (apparently healthy) patients (10/36) with other patients with comorbidities and/or immunosuppressed patients (26/36). Three other patients were excluded due to a lack of clinical data, or because the isolation of Cryptococcus spp. was considered as only colonization.

Clinical and epidemiological data were collected from patients' medical records, including age, sex, underlying diseases, and predisposing factors for cryptococcal disease. The involvement of organs and tissues by Cryptococcus spp. was assessed by clinical manifestations, radiographic images, the isolation site of this yeast, cerebrospinal fluid (CSF) analysis, and biopsy of the lung, skin, and lymph nodes. Antifungal treatment for meningitis and bloodstream infection was performed with deoxycholate amphotericin $\mathrm{B}$ $(\mathrm{CN}=11 / 17 ; \mathrm{CG}=4 / 7)$ or liposomal amphotericin $\mathrm{B}(\mathrm{CN}=6 / 17$; $\mathrm{CG}=3 / 7)$ and was maintained until there was no fungal growth in the CSF, and these drugs were associated or not with fluconazole. The consolidation and maintenance phases of the antifungal therapy were performed using fluconazole. Patients with lung and skin lesions and without meningitis were treated orally with fluconazole or itraconazole. The outcome was determined one year after diagnosis, and cases were classified as Cure-Improvement or Death.

Cryptococcus spp. were isolated from the following clinical samples: $\operatorname{CSF}(n=23)$, blood $(n=9)$, skin biopsy $(n=4)$, bronchoalveolar lavage $(\mathrm{n}=2)$, and a sample of each of the following materials: sputum, lung biopsy, pleural fluid, lymph node biopsy, and urine. Sabouraud dextrose agar with or without chloramphenicol was used to isolate the fungus, and Bact Alert (Biomérieux Brasil) or BD (Becton Dickinson and Company, USA) flasks were used for blood culture. Identification of the genus Cryptococcus was carried out by conventional laboratory methods of clinical mycology and/or the automated Vitek (BioMérieux Brasil) system. Clinical isolates of Cryptococcus spp. were maintained in the laboratory using periodic subcultures.

Molecular identification of $\mathrm{CN} / \mathrm{CG}$ species complexes was carried out by polymerase chain reaction (PCR) using pairs of specific primers that amplify DNA fragments to $695 \mathrm{bp}$ for C. neoformans (CNa-70a/CNa-70s) and 448 bp for C. gattii (CNb$49 \mathrm{a} / \mathrm{CNb}-49 \mathrm{~s})^{10}$. The molecular types of the CN (VNI, VNII, VNIII, and VNIV) and CG complexes (VGI, VGII, VGIII, and VGIV) were assessed by restriction fragment length polymorphism (RFLP) of the URA5 gene. After amplification, the products were subjected to enzymatic restriction with the restriction endonucleases HhaI (Invitrogen, Thermo Fisher) and Cfr13I (Invitrogen, Thermo Fisher) ${ }^{11,12}$. PCR-RFLP patterns were assigned visually by comparing them to the standard strains C. neoformans, molecular type VNI (WM148); C. neoformans, molecular type VNII (WM626); C. neoformans $\times C$. deneoformans hybrid, molecular type VNIII (WM628); C. deneoformans, molecular type VNIV (WM629); C. gattii, molecular type VGI (WM179); C. deuterogattii, molecular type VGII (WM178); C. bacillisporus, molecular type VGIII (WM175), and C. tetragattii, molecular type VGIV (WM779) from the Laboratory of Mycology (Pathogenic Fungi Collection) at the Oswaldo Cruz Foundation (FIOCRUZ)-INI/FIOCRUZ in Brazil.

The titer of the cryptococcal antigen in the CSF of 18 patients was determined by the latex agglutination method using the CALAS ${ }^{\circledR}$ Kit (Meridian Bioscience, USA). The titer of anti-Cryptococcus antibodies in the serum of 27 patients was measured by counterimmunoelectrophoresis using in-house prepared antigen, which were obtained by sonicating four samples of clinical isolates of $C$. neoformans (identified by cultivation in L-canavanine-glycine-bromothimol blue medium).

Statistical analysis was performed using GraphPad Prism v.6 (GraphPad Software, La Jolla, CA, USA). The proportions were compared using the chi-square test or Fisher's exact test. The MannWhitney U test was used to assess CSF parameters and the titer of the serum anti-Cryptococcus spp. antibody. The significance level was set at $P<0.05$.

The research project was approved by the Research Ethics Committee of University Hospital, Ribeirão Preto Medical School (No. 12247/2010).

\section{RESULTS}

Thirty-six cases of cryptococcosis in non-HIV-infected patients in southeastern Brazil were analyzed in this study. Among the patients, $72.3 \%(26 / 36)$ were individuals with comorbidities and/or immunosuppressed and $27.7 \%$ (10/36) were immunocompetent (apparently healthy) patients. Strains isolated from the $\mathrm{CN}$ group were $100 \%$ (24/24) of the VNI genotype (C. neoformans). The CG group consisted of $17 \%(2 / 12)$ of the VGI genotype (C. gattii sensu stricto) and $83 \%(10 / 12)$ of the VGII genotype (C. deuterogattii).

Age, sex, and associated conditions of the patients showed no significant differences between the $\mathrm{CG}$ and $\mathrm{CN}$ groups. The proportion of immunocompetent patients was higher in the CG group (Table 1). Patients from the CG group reported more 
TABLE 1: Demographic data, associated conditions, and predisposing factors according to the C. neoformans/C. gattii species complex.

\begin{tabular}{|c|c|c|c|c|}
\hline & $\begin{array}{c}\text { C. gattii* } \\
\mathrm{n} / \%\end{array}$ & $\begin{array}{c}\text { C. neoformans } \\
\mathrm{n} / \%\end{array}$ & $\begin{array}{l}\text { Total } \\
\text { n/\% }\end{array}$ & $P$ value ** \\
\hline Age - median (range) & $46.5(4-73)$ & $46.0(2.5-80)$ & $46.5(2.5-80)$ & 0.7355 \\
\hline Gender (Male: Female) & $10: 2$ & $16: 8$ & $26: 10$ & 0.6667 \\
\hline Associated Conditions & $7 / 58$ & $19 / 79$ & $26 / 72$ & 0.2474 \\
\hline Diabetes mellitus & $4 / 33$ & $4 / 17$ & $8 / 22$ & 0.3974 \\
\hline Malignancy ${ }^{a}$ & $0 / 0$ & $5 / 21$ & $5 / 14$ & 0.1494 \\
\hline Chronic visceral diseases ${ }^{b}$ & $6 / 50$ & $11 / 46$ & $18 / 50$ & 1.0 \\
\hline Systemic erythematosus lupus & $0 / 0$ & $2 / 8$ & $2 / 6$ & 0.5429 \\
\hline Kidney transplantation & $0 / 0$ & $3 / 13$ & $3 / 8$ & 0.5361 \\
\hline Non-associated conditions & $5 / 42$ & $5 / 21$ & $10 / 28$ & 0.2474 \\
\hline \multicolumn{5}{|l|}{ Predisposing factors } \\
\hline Environmental exposition ${ }^{c}$ & $10 / 83$ & $4 / 17$ & $14 / 39$ & 0.0002 \\
\hline Pharmacologic immunosuppression & $0 / 0$ & $6 / 25$ & $6 / 17$ & 0.0793 \\
\hline Alcoholism & $3 / 25$ & $3 / 13$ & $6 / 17$ & 0.3781 \\
\hline Malnutrition & $0 / 0$ & $3 / 13$ & $3 / 8$ & 0.5361 \\
\hline Patients - Total & $12 / 100$ & $24 / 100$ & $36 / 100$ & \\
\hline
\end{tabular}

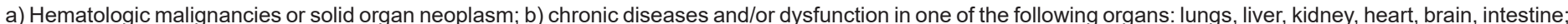
c) living or working in rural areas and/or regular contact with birds or wood; * C. gattii = C. deuterogattii $(\mathrm{n}=10)$, C. gattii s.s. $(\mathrm{n}=2)$; ${ }^{* *}$ statistical analysis.

prolonged exposure in a rural environment and/or working with wood or raising birds $(P=0.0002)$. Immunosuppression by corticosteroids or cytotoxic drugs was observed only in the $\mathrm{CN}$ group, while alcoholism and diabetes mellitus were observed in patients from both groups (Table 1).

Meningitis was the most common clinical manifestation in both of the groups. Cryptococcemia occurred only in the $\mathrm{CN}$ group $(P=0.0163)$, while a trend towards a higher proportion of lung and skin lesions, in addition to a patient with generalized lymphadenopathy, was observed in the CG group (Table 2). Patients in the two groups presented no differences in cellularity, glucose, protein, and cryptococcal antigen levels in the CSF. Patients in the CG group showed higher serum reactivity and slightly higher antibody titers against Cryptococcus spp. antigens (Table 2).

Patients in the $\mathrm{CN}$ and $\mathrm{CG}$ groups received similar antifungal treatments, except for the use of amphotericin B monotherapy in six patients in the $\mathrm{CN}$ group. Lethality was higher in the $\mathrm{CN}$ group $(48 \%)$ than in the CG group (18\%), without reaching statistical significance. Cure/improvement in the CG group was verified in 2/2 patients with C. gattii s.s. infection and in 5/7 cases of $C$. deuterogattii infection. The period between the diagnosis of cryptococcosis and death was significantly shorter in the $\mathrm{CN}$ group (Table 3). Sequelae occurred in three patients: in the CG group, a child had amaurosis and delay in neuromotor development, and another patient had hypoacusis. In the $\mathrm{CN}$ group, amaurosis occurred in one patient.

Table 4 compares cryptococcal disease in patients with the presence or absence of comorbidities or organ transplants.
Immunocompetent patients (absence of comorbidity or organ transplantation) had cryptococcosis caused by both $\mathrm{CG}$ and $\mathrm{CN}$ species complexes and a higher frequency of meningitis (90\%) $(P=0.0245)$. Lethality was higher in patients with comorbidities $(46 \%)$ than in immunocompetent patients $(20 \%)$, although the difference was not statistically significant (Table 4).

\section{DISCUSSION}

The cryptococcal disease of non-HIV-infected patients evaluated in this study was mainly related to the genotypes VNI of $\mathrm{CN}(C$. neoformans) and VGII of CG (C. deuterogattii), a finding similar to that observed in clinical isolates in Brazil ${ }^{6,13}$. The $\mathrm{CG}$ species complex has been associated with immunocompetent individuals ${ }^{14}$, but our study revealed that $C$. neoformans also infects such people, although they are more prevalent in comorbid or organ-transplanted patients. Immunocompetent individuals who are apparently healthy may have small defects in their immune capacity, facilitating cryptococcal infection ${ }^{15}$. In the studied cases, $72 \%$ of the patients had previously altered health conditions, and both patients infected with the $\mathrm{CN}$ and $\mathrm{CG}$ species complex had similar rates of chronic non-infectious diseases. The immunocompetent cases (42\%) and comorbidity rates in patients infected by the CG species complex were similar to those found in a large series of cases in Canada ${ }^{16}$. Only patients infected by $C$. neoformans had neoplasms and immunosuppression by corticosteroids or cytotoxic drugs, which suggests some specificity in the pathogenesis of the disease caused by different species of Cryptococcus. However, the disease caused by the CG species complex has already been associated with severe immunosuppression ${ }^{17}$. Diabetes mellitus and alcoholism are likely predisposing factors for the disease caused by both the $\mathrm{CN}$ and 
TABLE 2: Clinical and laboratory manifestations of the cryptococcal disease according to the C. neoformans/C. gattii species complex.

\begin{tabular}{|c|c|c|c|c|}
\hline & $\begin{array}{l}\text { C. gattii complex } \\
\qquad \mathrm{n} / \%\end{array}$ & $\begin{array}{c}\text { C. neoformans } \\
\mathrm{n} / \%\end{array}$ & $\begin{array}{l}\text { Total } \\
\mathrm{n} / \%\end{array}$ & $P$ value* \\
\hline \multicolumn{5}{|l|}{ Clinical Manifestation } \\
\hline Meningitis & $7 / 58$ & $15 / 63$ & $22 / 61$ & 1.0 \\
\hline Brain granuloma or pseudocyst & $3 / 25$ & $1 / 4$ & $4 / 11$ & 0.0980 \\
\hline Cryptococcemia & $0 / 0$ & $9 / 38$ & $9 / 25$ & 0.0163 \\
\hline Pulmonary lesion & $8 / 67$ & $9 / 38$ & $17 / 47$ & 0.1582 \\
\hline Cutaneous lesion & $3 / 25$ & $3 / 13$ & $6 / 17$ & 0.3781 \\
\hline Lymphadenopathy & $1 / 8$ & $0 / 0$ & $1 / 3$ & 0.3333 \\
\hline CSF alterations & $(n=7)$ & $(n=15)$ & $(n=22)$ & \\
\hline Cells - no./ $\mu \mathrm{L}-$ mean $\pm \mathrm{SD}$ & $100.42 \pm 11.99$ & $115.73 \pm 236.80$ & $110.8 \pm 80.05$ & 0.6867 \\
\hline Protein $-\mathrm{mg} / \mathrm{dL}-$ mean $\pm \mathrm{SD}$ & $161.28 \pm 236.80$ & $144.42 \pm 182.22$ & $150 \pm 196.1$ & 0.8582 \\
\hline Glucose - mg/dL - mean \pm SD & $45.85 \pm 45.38$ & $30.28 \pm 24.05$ & $35.4 \pm 32,4$ & 0.3115 \\
\hline Cryptococcal antigen titer ${ }^{\mathrm{a}}$ - median ange) & $\begin{array}{c}\geq 4096 \\
(64-\geq 4096)\end{array}$ & $\begin{array}{c}3072 \\
(N R-\geq 4096)\end{array}$ & $\begin{array}{c}\geq 4096 \\
(N R-\geq 4096)\end{array}$ & 0.4714 \\
\hline \multicolumn{5}{|l|}{ Antibodies anti-Cryptococcus in serumb } \\
\hline Reactive patients / total & $7 ; 11 / 64$ & $4 ; 16 / 25$ & $11 ; 27 / 41$ & 0.0608 \\
\hline Antibodies titer - median (range) & $8(1-16)$ & $2.5(1-8)$ & $4(1-16)$ & 0.0264 \\
\hline Patients - Total & $12 / 100$ & $24 / 100$ & $36 / 100$ & \\
\hline
\end{tabular}

a) Latex agglutination test: titer-inverse of CSF dilution; b) counterimmunoelectrophoresis test: titer-inverse of serum dilution; *statistical analysis; SD: standard deviation; CSF: cerebrospinal fluid; NR: non-reactive.

TABLE 3: Antifungal drug treatment and outcome of patients with cryptococcosis due to the C. neoformans/C. gattii species complex.

\begin{tabular}{|c|c|c|c|c|}
\hline & $\begin{array}{c}\text { C. gattii complex } \\
\mathrm{n} / \%\end{array}$ & $\begin{array}{c}\text { C. neoformans } \\
n / \%\end{array}$ & $\begin{array}{l}\text { Total } \\
\mathrm{n} / \%\end{array}$ & $p$ value $^{*}$ \\
\hline \multicolumn{5}{|l|}{ Antifungal treatment } \\
\hline Amphotericin B $\rightarrow$ Fluconazole & $4 / 33^{a}$ & $5 / 21$ & $9 / 25$ & 0.4428 \\
\hline Amphotericin B + Fluconazole & $3 / 25$ & $6 / 25$ & $9 / 25$ & 1.0 \\
\hline Amphotericin B & $0 / 0$ & $6 / 25$ & $6 / 17$ & 0.0793 \\
\hline Fluconazole & $4 / 33$ & $3 / 13$ & $7 / 19$ & 0.1904 \\
\hline Itraconazole & $0 / 0$ & $1 / 4$ & $1 / 3$ & 1.0 \\
\hline No antifungal & $1 / 8$ & $3 / 13$ & $4 / 11$ & 1.0 \\
\hline \multicolumn{5}{|l|}{ Outcome } \\
\hline Cure/improvement ${ }^{\mathrm{b}}$ & $9 / 82$ & $12 / 52$ & $21 / 62$ & 0.1398 \\
\hline Death $^{\mathrm{b}}$ & $2 / 18$ & $11 / 48$ & $13 / 38$ & \\
\hline \multicolumn{5}{|l|}{ Diagnosis - death time (days) } \\
\hline median (range) & $53(31-75)$ & $7(2-79)$ & $12(2-79)$ & 0.0803 \\
\hline Unknown & 1 & 1 & 2 & \\
\hline Sequels & $2 / 17$ & $1 / 4$ & $3 / 8$ & 0.2527 \\
\hline Patients - Total & $12 / 100$ & $24 / 100$ & $36 / 100$ & \\
\hline
\end{tabular}

a) One patient used ketoconazole instead of fluconazole; b) survival and lethality rates excluded patients whose outcome is unknown; ${ }^{*}$ statistical analysis. 
TABLE 4: Cryptococcal disease and outcome of patients with or without comorbidities and organ transplantation, regardless of the Cryptococcus species.

\begin{tabular}{|c|c|c|c|}
\hline & \multicolumn{2}{|c|}{ Comorbidity or organ transplantation } & \multirow[t]{2}{*}{$P$-value* } \\
\hline & Absent $\mathrm{n} / \%$ & Present $\mathrm{n} / \%$ & \\
\hline \multicolumn{4}{|c|}{ Cryptococcus species complex } \\
\hline C. gattii complex & $5 / 50$ & $7 / 27$ & 0.2474 \\
\hline C. neoformans & $5 / 50$ & $19 / 73$ & \\
\hline \multicolumn{4}{|l|}{ Cryptococcosis site } \\
\hline Meningitis & $9 / 90$ & $12 / 46$ & 0.0245 \\
\hline Cryptococcemia & $1 / 10$ & $8 / 31$ & 0.3921 \\
\hline Pulmonary & $2 / 20$ & $14 / 54$ & 0.1326 \\
\hline Cutaneous & $1 / 10$ & $5 / 19$ & 0.6546 \\
\hline Lymphadenopathy & $0 / 0$ & $1 / 4$ & 1.0 \\
\hline \multicolumn{4}{|l|}{ Outcome $^{a}$} \\
\hline Cure/improvement & $8 / 80$ & $13 / 54$ & 0.2508 \\
\hline Death & $2 / 20$ & $11 / 46$ & \\
\hline Unknown & 0 & 2 & - \\
\hline Patients - Total & $10 / 100$ & $26 / 100$ & \\
\hline
\end{tabular}

Cure/improvement and death rates excluded patients whose outcome was unknown; *statistical analysis.

CG species complexes ${ }^{18,19}$. Exposure to environmental sources that contain Cryptococcus spp. was associated with C. gattii infection, similar to that observed in Australia ${ }^{7}$, in which many patients lived in rural areas. The CG group included a child with cryptococcal meningitis caused by C. deuterogattii (VGII genotype) after returning from a trip to a CG species complex endemic area in northeastern Brazil. C. gattii s.s. was isolated from a patient who caught wild birds and presented with Moyamoya disease and generalized cryptococcal lymphadenopathy. C. gattii s.s. was also isolated from an immunocompetent man who presented with a chronic cutaneous ulcer and had past contact with house birds.

Meningitis and disseminated disease are the most common clinical manifestations in non-HIV-infected patients, but CG species can lead to a predominance of lung involvement or cause primary skin lesions ${ }^{9,20}$. The frequency of meningeal, lung, and skin lesions showed no difference between the CN and CG group. A significant difference was found in bloodstream infection, which occurred only in patients infected with $C$. neoformans. This is probably a consequence of more severe immunosuppression in patients in the $\mathrm{CN}$ group, facilitating fungal dissemination. The frequency of patients with cryptococcal fungemia was higher in this investigation (25\%) than in other Brazilian report of cryptococcosis in non-HIV-infected and non-transplanted patients $(6.8 \%)^{21}$. This lower percentage is probably due to the high proportion of immunocompetent patients and CG complex infections in the later study.

The higher level of serum anti-Cryptococcus antibodies among patients infected by CG could be related to the lower frequency of immunosuppression in the patients in this group. A previous study found a higher humoral response of IgG and IgA antibodies in patients infected with $C$. gattii than in those infected with C. neoformans ${ }^{22}$.
The outcome of antifungal treatment with amphotericin B and/or azole drugs showed a trend towards lower lethality among patients infected by CG species. The overall lethality in patients in this study (38\%) was higher than the $21 \%$ lethality found in another series of cryptococcosis cases in non-HIV-infected patients in Brazil, although that study had a higher percentage of immunocompetent individuals ${ }^{21}$. The death of patients at the beginning of antifungal treatment has been observed in other hospitals and has been associated with cryptococcemia and high lactate levels in $\operatorname{CSF}^{23,24}$. Patients with cryptococcal disease due to CG species showed a lethality after 12 months which reached 18\% in this study, 23.3\% in a British Colombia-Canada study, and was more elevated in some series of cases that included immunosuppressed individuals and children ${ }^{16,25,26}$.

The comparison of clinical manifestations possibly attributable to the type of causative agent, $\mathrm{CN}$ or CG complexes, may have been impaired by the higher proportion of immunosuppressed patients in the $\mathrm{CN}$ group. Thus, the clinical picture and outcome were compared between immunocompetent patients and those with previous diseases and/or immunosuppression, regardless of the Cryptococcus species. Cryptococcal meningitis was most commonly seen among immunocompetent patients, with a trend for cryptococcemia and pulmonary involvement manifested mainly in cases with comorbidities and/or immunosuppression. The high proportion of clinically expressed meningitis may be a consequence of the high immunological reactivity of immunocompetent patients. Tissue injury and damage in cryptococcosis are more likely to occur when the immune response of the host is very weak or very intens $\mathrm{e}^{27}$. Immunocompetent patients also had lower lethality, although the difference was not statistically significant. HIV-infected patients with controlled or active disease (AIDS) 
showed similar lethality among those infected with $C$. gattii or non-C. gattii species ${ }^{28}$. Such data suggest that not only the Cryptococcus species but also the health condition and immunological capacity of the host are important in defining the clinical presentation and outcome of patients ${ }^{14}$.

This study was limited by the small number of cases, making it difficult to differentiate patient groups based on the analyzed parameters. Other cases of cryptococcosis in non-HIV-infected patients were recognized at the institution during the same period, but without the availability of isolated microorganisms for genotyping.

In conclusion, cryptococcal disease caused by $C$. neoformans (VNI genotype) was associated with immunodepressed patients and fungemia, and patients infected with $C$. deuterogattii and C. gattii s.s. (genotypes VGII and VGI of CG) were exposed to environmental sources of Cryptococcus spp. and showed a higher humoral immune response. Chronic non-infectious diseases, diabetes mellitus, and alcoholism were likely predisposing factors for infection by both $\mathrm{CN}$ and $\mathrm{CG}$ species. Immunocompetent patients (without comorbidity or solid organ transplantation) showed a high incidence of cryptococcal meningitis, a trend toward less fungal dissemination, and longer patient survival, regardless of the infecting species. The clinical expression and outcome of cryptococcal disease in non-HIV-infected patients are probably more related to the health and immunological conditions of the host than to the Cryptococcus species complexes.

\section{AUTHORS' CONTRIBUTION}

EN: Conception and design of the study, Acquisition of data, Analysis and interpretation of data, Final approval of the version to be submitted; PHGB: Analysis and interpretation of data; MRVZK: Analysis and interpretation of data, Final approval of the version to be submitted; FCV: Conception and design of the study, Acquisition of data; RCS: Conception and design of the study, Acquisition of data; GGG: Conception and design of the study, Acquisition of data RM: Conception and design of the study, Acquisition of data, Analysis and interpretation of data, Final approval of the version to be submitted.

\section{FINANCIAL SUPPORT}

Erika Nascimento was the recipient of a fellowship from Fundação de Amparo à Pesquisa do Estado de São Paulo (FAPESP) for the execution of this study (Protocol FAPESP 2010/519322), the Coordenação de Aperfeiçoamento de Pessoal de Nível Superior - (CAPES Brazil - Finance Code 001) for by granting postdoctoral scholarships Grant numbers: 88882.317609/2019-1, and the Fundação de Apoio ao Ensino, Pesquisa e Assistência do Hospital das Clínicas da Faculdade de Medicina de Ribeirão Preto da Universidade de São Paulo (FAEPA) for financial support.

\section{CONFLICT OF INTEREST}

The authors declare that there is no conflict of interest.

\section{ORCID}

Erika Nascimento: 0000-0002-4141-7414

Patrícia Helena Grizante Barião: 0000-0003-1335-3975

Marcia Regina von Zeska Kress: 0000-0003-1239-7722

Fernando Crivelenti Vilar: 0000-0001-8232-5375

Rodrigo de Carvalho Santana: 0000-0002-5887-8663

Gilberto Gambero Gaspar: 0000-0002-6804-454X

Roberto Martinez: 0000-0001-6906-7981

\section{REFERENCES}

1. Chang CC, Chen SC. Colliding epidemics and the rise of cryptococosis. J Fungi (Basel). 2015;2(1):1.

2. Gassiep I, Douglas J. Emeto TI, Crawley K, Playford EG. Cryptococcal infections over a 15 year period at a tertiary facility \& impact of guideline management. Mycoses. 2018;61(9):633-6.

3. Hevey M, George IA, Raval K, Powderly WG, Spec A. Presentation and mortality of cryptococcal infection varies by predisposing illness, a retrospective cohort study. Am J Med. 2019;132(8):977-83.

4. Halloran JA, Powderly WG, Spec A. Cryptococcosis today: It is not all about HIV infection. Curr Clin Microbiol Rep. 2017;4(2):88-95.

5. Kwon-Chung KJ, Bennett JE, Wickes BL, Meyer W, Cuomo CA, Wellenburg KR, et al. The case for adopting the "species complex", nomenclature for the ethiologic agents of cryptococcosis. mSphere. 2017;2(1):e00357-16.

6. Trilles L, Lazéra M dos S, Wanke B, Oliveira RV, Barbosa GG, Nishikawa MM, et al. Regional pattern of the molecular types of Cryptococcus neoformans and Cryptococcus gattii in Brazil. Mem Inst Oswaldo Cruz. 2008;103(5):455-62.

7. Mitchell DH, Sorrell TC, Allworth AM, Heath $\mathrm{CH}$, McGregor AR, Papanaoum K, et al. Cryptococcal disease of CNS in immunocompetent hosts: influence of cryptococcal variety on clinical manifestations and outcome. Clin Infect Dis. 1995; 20(3):611-6.

8. Pinheiro SB, Souza ES, Cortez ACA, da Silva Rocha DF, Menescal LSF, Chagas VS, et al. Cryptococcal meningitis in non- HIV patients in the State of Amazonas, Northern Brazil. Braz J Microbiol. 2021;52(1):279-88.

9. Chen SC, Meyer W, Sorrell TC. Cryptococcus gattii infections. Clin Microbiol Rev. 2014;27(4):980-1024.

10. Aoki FH, Imai T, Tanaka R, Mikami Y, Taguchi H, Nishimura NF, et al. New PCR primer pairs specific for Cryptococcus neoformans serotype A or B prepared on the basis of random amplified polymorphic DNA fingerprint pattern analyses. J Clin Microbiol.1999;37(2):315-20.

11. Meyer W, Castañeda A, Jackson S, Huynh M, Castañeda E, Ibero American Cryptococcal Study Group. Molecular typing of Iberoamerican Cryptococcus neoformans isolates. Emerg Infect Dis. 2003;9(2):189-95.

12. Meyer W, Trilles L. Genotyping of the Cryptococcus neoformans/ C. gattii species complex. Aust Bioc. 2010;41(1):12-6.

13. Firacative C, Meyer W, Castañeda E. Cryptococcus neoformans and Cryptococcus gattii species complexes in Latin America: a map of molecular types, genotypic diversity, and antifungal susceptibility as reported by the Latin American Cryptococcal Study Group. J Fungi (Basel). 2021;7(4):282.

14. Chen S, Sorrell T, Nimmo G, Speed B, Currie B, Ellis D, et al. Epidemiology and host-and variety-dependent characteristics of infection due to Cryptococcus neoformans in Australia and New Zealand. Australasian 
Cryptococcal Study Group. Clin Infect Dis. 2010;31(2):499-508.

15. Brizendini KD, Baddley JW, Pappas PG. Predictors of mortality and diferences in clinical features among patients with cryptococcosis according to imune status. Plos One. 2013;8(3):e60431.

16. Phillips P, Galanis E, Mc Dougall L, Chong MY, Balshaw R, Cook VJ, et al. Longitudinal clinical findings and outcome among patients with Cryptococcus gattii infection in British Columbia. Clin Infect Dis. 2015;60(9):1368-76.

17. MacDougall L, Fyfe M, Romney M, Starr M, Gallanis E. Risk factors for Cryptococcus gattii infection, British Columbia, Canada. Emerg Infect Dis. 2011; 17(2):193-9.

18. Liu KH, Chen CM, Chen TL, Kuo SC, Kao CC, Jeng YC, et al. Diabetes mellitus is associated with aquisicion and increased mortability in HIVuninfected patients with cryptococcosis: A population - based study. $\mathrm{J}$ Infect. 2016;72(5):608-14.

19. Hou X, Kou L, Han X, Zhu R, Sang L, Liu T. Pulmonary cryptococcosis characteristics in immunocompetent patients - A 20-year clinical retrospective analysis in China. Mycoses. 2019;62(10):937-44.

20. Nascimento E, Bonifácio da Silva ME, Martinez R, von Zeska Kress MR. Primary cutaneous cryptococcosis in an immunocompetent patient due to Cryptococcus gattii molecular type VGI in Brazil: a case report and review. Mycoses. 2014;57(7):442-7.

21. Lomes NR, Melhem MS, Szeszs MW, Martins M dos A, Buccheri R. Cryptococcosis in non-HIV/ non-transplant patients: a Brazilian case series. Med Mycol. 2016;54(7):699-706.

22. Speed BR, Kaldr J, Cairns B, Pegorer M. Serum antibody response to active infection with Cryptococcus neoformans and its varieties in immunocompetent subjects. J Med Vet Mycol. 1996;34(3):187-93.

23. Tsai WC, Lien CY, Lee JJ, Huang CR, Tsai NW, Chang CC, et al. The clinical characteristics of adult cryptococcal meningitis patients who died within one year of treatment with a focus on those with early mortality. J Clin Neurosci. 2019;67:80-4.

24. Ponzio V, Chen Y, Rodrigues AM, Tenor JL, Toffaletti DL, MedinaPestana JO, et al. Genotypic diversity and clinical outcome of cryptococcosis in renal transplant recipientes in Brazil. Emerg Microbes Infect.2019;8(1):119-29.

25. Damasceno-Escoura AH, de Souza ML, de Oliveira Nunes F, Pardi TC, Gazotto FC, Florentino DH, et al. Epidemiological, clinical and outcome aspects of patients with cryptococcosis caused by Cryptococcus gattii from a non-endemic area of Brazil. Mycopathologia. 2019;184(1): $65-71$.

26. Martins LM, Wanke B, Lazéra M dos S, Trilles L, Barbosa GG, Macedo $\mathrm{RC}$, et al. Genotypes of Cryptococcus neoformans and Cryptococcus gattii as agentes endemic cryptococcosis in Terezina, Piaui (northeastern Brazil). Mem Inst Oswaldo Cruz. 2011;106(6):725-30.

27. Pirofski LA, Casadevall A. Immune-mediated damage completes the parábola: Cryptococcus neoformans pathogenesis can reflect the outcome of a weak or strong immune response. mBio. 2017;8(6): e02063-17.

28. Morgan J, McCarthy KM, Gould S, Fan K, Arthington-Skaggs B, Iqbal $\mathrm{N}$, et al. Cryptococcus gattii infection characteristics and epidemiology of cases identified in a South African Province with high HIV seroprevalence, 2002-2004. Clin Infect Dis. 2006;43(8):1077-80. 